\title{
Success of Instant-Start Life-Saving Peritoneal Dialysis in Patients with End- Stage Vascular Access Failure: Time to Rescue Patients
}

\author{
Abdullah K Al-Hwiesh ${ }^{1, *}$, Ibrahiem Saeed Abdul-Rahman ${ }^{1}$, Nadia Al-Audah', Amani Al-Hwiesh ${ }^{1}$, Abdulrahman Taha ${ }^{3}$, \\ Mohammed A Nasr El-Din ${ }^{1}$, Nehad Al-Audah ${ }^{1}$, Abdalla Abdelrahman ${ }^{4}$, Ali Harbi ${ }^{5}$, Muaz M Abdel Galil ${ }^{1}$, Dujanah Mousa ${ }^{5}$, Habib \\ Al-Ramadan ${ }^{1}$, Khalid Al-Otaibi ${ }^{6}$, Sami Skhiri ${ }^{5}$, Dalal AlKhaldi ${ }^{5}$, and Jose Alberto Scapuzzi ${ }^{5}$
}

${ }^{1}$ Nephrology Division, Department of Internal Medicine, King Fahd Hospital of the University, Imam Abdulrahman Bin Faisal University, Saudi Arabia

${ }^{2}$ Department of Pathology, Dammam Central Hospital, Dammam, Saudi Arabia

${ }^{3}$ Department of Computer signs, AFDA, Cape Town, South Africa

${ }^{4}$ Department of Electrical Engineering, Queen's University, Kingston, Canada

${ }^{5}$ Diaverum Dialysis Centers, Eastern Province, Saudi Arabia

${ }^{6}$ Department of Urology, King Fahd Hospital of the University, Imam Abdulrahman Bin Faisal University, Saudi Arabia

*Corresponding author: Abdullah Al-Hwiesh, Professor and Consultant of Nephrology, Nephrology Division, Department of Internal Medicine, King Fahd Hospital of the University, Imam Abdulrahman Bin Faisal University, Saudi Arabia, E-mail: ahwiesh@iau.edu.sa

Received: 21 Aug, 2019 | Accepted: 16 Sep, 2019 | Published: 23 Sep, 2019

Citation: Al-Hwiesh AK, Abdul-Rahman IS, Al-Audah N, Al-Hwiesh A, Taha A, et al. (2019) Success of Instant-Start Life-Saving Peritoneal Dialysis in Patients with End-Stage Vascular Access Failure: Time to Rescue Patients. Int J Nephrol Kidney Fail 5(4): dx.doi.org/10.16966/23805498.182

Copyright: (c) 2019 Al-Hwiesh AK, et al. This is an open-access article distributed under the terms of the Creative Commons Attribution License, which permits unrestricted use, distribution, and reproduction in any medium, provided the original author and source are credited.

\begin{abstract}
Background: This study aimed at evaluation of the visibility of instant-start, life-saving peritoneal dialysis in dialysis patients with End Stage Vascular Access Failure (ESVAF) in addition to complications and outcome of this dialysis modality.

Methods: This is a prospective observational study from June 2015 throughout July 2018. Urgent two-cuff curled Tenckhoff PD catheters were inserted percutaneously within few hours by expert nephrology team. Twenty-three dialysis ESVAF patients who presented acutely to our hospital were included in the study.

Results: Dialysis was initiated within 2 hours (range: 1-3 h) from presentation in all patients. Adequate metabolic and volume control was achieved after 4 sessions of PD. Mechanical complications occurred in $25.1 \%$. The main mechanical complications were minor peri-catheter leakage (13\%) and catheter tip migration (8.7\%). One patient had omental wrap which necessitated catheter replacement. Exit-Site Infection (ESI) occurred in $13 \%$, tunnel infection in $0 \%$ and peritonitis in $13 \%$ of patients. Peritonitis rate was 0.142 episode/patient-year. After 6 months, catheter and patient survival rates were $87.0 \%$ and $91.3 \%$ respectively.
\end{abstract}

Conclusion: The instant-start PD modality was a life-saving, feasible and safe alternative to hemodialysis in patients with ESVAF.

Keywords: ESVAF; PD; Catheter insertion; Mechanical complications; Infectious complications; Outcomes

\section{Introduction}

Hemodialysis is the most common dialysis modality worldwide. Good functioning Vascular Access (VA) is the Achilles heel for efficient and successful hemodialysis [1,2]. Up to $30 \%$ of admissions in hemodialysis population is related to vascular access dysfunction, leading to increase in morbidity and mortality [3]. Vascular access dysfunction constitutes quarter of total of End Stage Renal Disease (ESRD) expenditure in the United States [4]. About $80 \%$ of incident ESRD patients started urgent hemodialysis through Central Venous Catheter (CVC) [5]. The high morbidity and mortality rates in patients with CVC may be related to catheter infection, catheter related septicemia, frequent admissions, inadequate dialysis related to insufficient blood flow and frequent interruption of dialysis due to venous stenosis or thrombosis [6-8]. Almasri J, et al. conducted a recent systematic review and meta-analysis of 200 studies. Of 875,269 vascular accesses, the primary patency at two years was higher for fistulas than for graft and catheters (55\%,50\% and 40\% respectively). The risk factors for lower patency rates were old age, female gender, diabetes and coronary artery disease. The two-year mortality was higher with catheters, followed by grafts and fistulas (26\%, 17\% and $15 \%$ 
respectively) [9]. Adapting peritoneal PD first had several advantages: preservation of residual renal function, survival advantages in the first two years, better quality of life and lower cost [10-13]. Unfortunately, still PD is underutilized worldwide. Reasons for PD underutilization include peritonitis complication, ultrafiltration failure, catheter related complications and improper predialysis education $[12,13]$. In Saudi Arabia, peritoneal dialysis is still underutilized as it constitutes $10 \%$ only of the total dialysis population. The reasons for underutilizations from patients' perspectives were elegantly highlighted by Dahlan RA, et al. as she pointed at lack of predialysis education programs and patients counseling, inability to start urgent peritoneal dialysis in some centers and the limited nephrologists' experience with PD catheter insertions [14]. As the number of hemodialysis patients increases, nephrologists will encounter more patients with End-Stage Vascular Access Failure (ESVAF) [15]. Unfortunately, there is no consensus on a precise definition of ESVAF. Al Shakarchi J, et al. [7] reported anatomical-based classification reflecting the degree of severity of access failure. ESVAF was defined as bilateral venous occlusion or severe stenosis which does not allow creation of a standard vascular access. Recently, urgent start peritoneal dialysis has started to gain popularity among nephrologists, thanks to the emergence of dedicated peritoneal dialysis centres sharing their successful programs and clinical data [15-20]. However, till the present time there is no single study addressing instant-start PD as a lifesaving modality where both physician and patient had no other options except for PD. Our patients' cohort had ESVAF while on HD due to exhaustion of all possible vascular accesses and haemodialysis was not an option any more. Patients were referred to our center for urgent start peritoneal dialysis. The aim of the current study was to address the feasibility of starting PD within few hours of presentation as a lifesaving modality in patients whom vascular accesses were completely exhausted and to look at patients' outcome.

\section{Methods}

This is a prospective observational study that was conducted from June 2015 through July 2018 at King Fahd University Hospital, Saudi Arabia in collaboration with Diaverum Hemodialysis Centers at Eastern Province of Saudi Arabia. The study was carried out in accordance with Helsinki's Declaration and was approved by King Fahd Hospital Human Ethical committee. Written informed consents were obtained from all patients.

Patients were referred to our center due to ESVAF defined by inability to create vascular access in both upper and lower limbs due to venous stenosis or thrombosis as documented by computer tomography or angiography. The average number of failed vascular access was 7-10 procedure for each patient. Urgent two-cuff curled Tenckhoff PD catheters were inserted percutaneously by expert nephrology team using Al-Hwiesh technique [21,22]. Tidal automated peritoneal dialysis was started instantly (average 2 hours) with 10 liters Physioneal 1.36 and 15 liters Physioneal 2.27\%. Each fill volume was 1.5 liter that was gradually increased to 2 liters over 7-10 days. All patients received first generation cephalosporin intravenously prior to the procedure. After hospital discharge, patients were followed-up daily with automated PD until patient or the care giver completes the course of PD training.

\section{PD catheter insertion technique}

Antibiotic prophylaxis was given as recommended, with first generation cephalosporin administered intravenously two hours prior to the procedure. The PD catheter was inserted under fluoroscopic guidance by the nephrologist in the day surgery room with the patient under local anesthesia and parenteral sedation (all patients received intravenous midazolam and $1 \%$ lignocaine). We used blind placement based on the Seldinger technique. A $2 \mathrm{~cm}$ paramedian incision was made 1-2 cm below the umbilicus, followed by blunt dissection of the subcutaneous tissue with an artery forceps until the fascia anterior to the rectus muscle was reached. The peritoneum was then punctured using a 16-gauge needle. A guidewire (straight, $120 \mathrm{~cm}$ long and $2 \mathrm{~mm}$ thick) was inserted into the peritoneal cavity through the 16-gauge needle. The needle was then removed leaving the guidewire in place. A hollow dilator (20-gauge) was introduced over the guidewire and used to dilate the passage (larger dilators can be used if necessary). The PD catheter was then inserted over the guidewire, overlapping and surrounding the hollow introducer [without the peel-away sheath and directed toward the Douglas pouch. The guidewire was then removed. Finally, the PD catheter was gradually advanced towards its goal, while the hollow introducer was removed. A subcutaneous tunnel was created using an artery forceps, and the PD catheter was adjusted through it. The inner cuff of the PD catheter was secured with a purse string suture on the fascia anterior to the rectus muscle. The original incision was then closed and the PD catheter was flushed several times with a total of $2 \mathrm{~L}$ heparinized $0.9 \%$ saline $(500 \mathrm{~mL}$ each fill) to confirm catheter patency and to check for intra-abdominal bleeding.

\section{Data and statistical analysis}

All the relevant data were obtained from the patient hemodialysis and radiology records and the hospital blood result computer database. Statistical analysis was performed using SPSS software (SPSS Inc., Chicago, IL; version 20). Results are shown as median and Inter-Quartile Range (IQR) or mean \pm SD and $p$ value was considered significant at $<0.05$.

\section{Results}

The demographic characteristics and patients' outcome are summarized in table 1 and figure 1. Between June 2015 throughout July 2018 we encountered 23 ESRD patients who were treated with hemodialysis and in whom all possible vascular accesses were exhausted (Table 2). At presentation 9 (39.1\%) patients had pulmonary congestion, $13(56.5 \%)$ with uremic manifestations, 6 (26.1\%) had electrolyte disturbances, and 4 (17.4\%) had pericarditis with or without pericardial effusion (Table 1). PD was started instantly after PD catheter insertion with gradually increasing fill volume. All insertions were done percutaneously by the nephrology team. Dialysis was initiated urgently within 2 hours (range1-3 h) from presentation in all patients.

Adequate metabolic and volume control was achieved after 4 sessions of PD. Mechanical complications occurred in $25.1 \%$. The main mechanical complications were minor peri-catheter leakage (3 patients-13\%) and catheter tip migration (2 patients-8.7\%). One patient had omental wrap which necessitated catheter replacement. Minor peri-catheter leakage of dialysate was managed by stopping the treatment for $24 \mathrm{~h}$ and restarting with a lower infusion volume. There was no need for catheter replacement due to leakage and no patient changed the dialysis method for this reason, and within approximately one week the infusion volume was back to $2 \mathrm{~L}$ per cycle. ESI occurred in $13 \%$ of patients, tunnel infection in $0 \%$, peritonitis in $13 \%$ (treated conservatively), and peritonitis rate was 0.142 episode/patient-year. After 6 months, catheter and patient survival rates were $87 \%$ and $91.3 \%$ respectively (Figure 2). Causes of death were cardiovascular event in one and cerebrovascular accident in another (4.3\%, each). There were no deaths associated with catheter implantation or PD therapy directly. Recovery of electrolyte balance occurred in 19 (82.6\%) patient 
Table 1: Demographic characteristic of the study patients.

\begin{tabular}{|c|c|}
\hline Age, median (IQR) & $47(35-58)$ \\
\hline Female/Male gender & $14 / 9$ \\
\hline BMI, mean & $26.8 \pm 2.5$ \\
\hline \multicolumn{2}{|l|}{ Comorbidities, number (\%) } \\
\hline - $\quad \mathrm{DM}$ & $10(43.5)$ \\
\hline - $\quad$ HTN & $14(60.9)$ \\
\hline - $\quad$ IHD & $4(17.4)$ \\
\hline - $\quad$ Heart failure & $3(13.0)$ \\
\hline - Dyslipidemia & $8(34.8)$ \\
\hline - $\quad$ SLE & $4(17.4)$ \\
\hline - $\quad$ CVA & $2(8.7)$ \\
\hline - $\quad$ Failed kidney transplant & $2(8.7)$ \\
\hline \multicolumn{2}{|l|}{ Patients' presentation, number (\%) } \\
\hline - Pulmonary congestion & $9(39.1)$ \\
\hline - Uremic features & $13(56.5)$ \\
\hline - Electrolyte disturbance & $6(26.1)$ \\
\hline - $\quad$ Pericarditis & $4(17.4)$ \\
\hline Number of failed vascular access (range) & $7-10$ \\
\hline Duration on HD, months Mean \pm SD & $20 \pm 11.7$ \\
\hline $\begin{array}{l}\text { Time from presentation to catheter } \\
\text { insertion, hours, median (IQR) }\end{array}$ & $2(1-3)$ \\
\hline Duration on PD, months Median (IQR) & $11(9-29)$ \\
\hline Mortality on PD, n (\%) & $2(8.7)$ \\
\hline
\end{tabular}

IQR: InterQuartile Ratio; DM: Diabetes Mellitus; HTN: Hypertension; IHD: Ischemic Heart Disease; SLE: Systemic Lupus Erythematosus; CVA: CerebroVascular Accident; HD: HemoDialysis; PD: Peritoneal Dialysis

Table 2: Technical and infectious complications.

\begin{tabular}{|l|c|l|c|}
\hline $\begin{array}{l}\text { Technical } \\
\text { complications }\end{array}$ & Number (\%) & $\begin{array}{c}\text { Infectious } \\
\text { complications }\end{array}$ & Number (\%) \\
\hline Failure of insertion & $0(0)$ & Exit-site infection & $3(13.0)$ \\
\hline Bowel perforation & $0(0)$ & Tunnel infection & $0(0 \%)$ \\
\hline Migration & $2(8.7)$ & Peritonitis & $3(13 \%)$ \\
\hline Omental wrap & $1(4.3)$ & & \\
\hline Early leakage* & $3(13)$ & & \\
\hline $\begin{array}{l}\text { Overall Catheter } \\
\text { survival }\end{array}$ & $20(87 \%)$ & & \\
\hline
\end{tabular}

*Early leakage was defined as leakage in the first week after starting PD.

after the fourth dialysis session. Four patients underwent kidney transplantation within the study period (Tables 3,4 ).

\section{Discussion}

In this study, we offered PD as a life-saving method for those patients with ESVAF. PD was initiated right within 1-3 hours of presentation. Our strategy was feasible and safe. The complication rate in our cohort was low and on aligns with that of previous reports in the field.
With the increased longevity of HD candidates, a cohort of patients is in the precarious position of having exhausted the standard routes of vascular access. The numbers of patients who have exhausted definitive access options will continue to increase and the extent of this problem of failed access or 'desperate measures' access is difficult to determine. A small percentage of patients will maintain autologous AVFs long-term, but for many patients a sequence of fistula and/or graft formations with subsequent interventions and ultimate failures will eventually result in standard options being exhausted; the so called ESVAF. The criteria determining this point are ill defined and vary between surgeons and units [23]. Despite the increasing prevalence of hemodialysis patients with complex access issues, there remains no consensus on the definition of ESVAF. An extreme definition of ESVAF would be whereby all potential routes of access have been utilized and hemodialysis [7] is no longer achievable, all of our patients fulfilled this definition.

Infection is the second most common cause of admission of dialysis patients after cardiovascular disease, the rates of admissions for catheter related septicemia are higher in HD patient reaching 102 per 1000 patient-year i.e., 1.4-2.3 times higher than that seen in PD patients leading to frequent hospitalization and increased mortality rates [24].

Unfortunately, PD is still underutilized worldwide. Factors behind this underutilization include risk of peritonitis, ultrafiltration failure, catheter related complications, improper predialysis education, in addition to the Increasing number of HD centers and physicians' comfort with this modality $[12,13]$. In United States alone PD has declined from $15 \%$ in 1980 to about $7 \%$ recently [12]. The rate of PD technique failure varied between $12 \%$ and $25 \%$ in different reports [10,25-29]. Causes of failure were catheter related complications, previous history of $\mathrm{HD}$, center size, assisted $\mathrm{PD}$ and early peritonitis episodes [26]. Similar results have been reported from Switzerland and Australia $[10,28,29]$. Our study, in contrast, did not show previous $\mathrm{HD}$ as a risk factor. In addition, control of the other risk factors has significantly minimized the rate of technique failure. The alleged correlation between $\mathrm{HD}$ and technique failure may be attributed to late referral of patients to the PD program with inadequate predialysis preparation and the subsequent loss of residual renal function.

As the numbers of patients who have exhausted definitive access continue to increase and patients with life threatening presentations continue to expand, the idea of starting the so called "urgent-use PD" gained popularity. Three previous studies assessed the risk of catheter dysfunction in urgent-start PD [30-32] and that varied from 1.9 to $33.3 \%$. These studies also assessed the rate of PD-related infections. The duration of assessment ranged from 1 to 6 months; peritonitis occurred in $2.4-15.4 \%$ and ESI ranged from 1.3 to $11 \%$. However, in those studies, the technique failure was similar in both urgent and conventional start PD. In our study, the rate of technique survival was $87 \%$ at 180 days, and ESI/peritonitis rates were within the internationally accepted ranges.

There is paucity of data in literature regarding the role of PD in those with ESVAF. Gameiro J, et al. reported a retrospective analysis of 30 patients with ESVAF over 11 years. Twenty of their patients were referred to $\mathrm{PD}$; their mean survival was $60 \pm 28.4$ months. Death rate was $30 \%$ and $60 \%$ of the death was in the first year after commencing dialysis [20]. In another study, Povlsen J, et al. reported a $7.7 \%$ incidence of leak in their surgically placed catheters using small fill volumes which was not significantly different from the incidence of catheter leak in their chronic PD patients; peritonitis rates were also similar [16]. See EJ, et al. [17] reported an incidence of catheter 


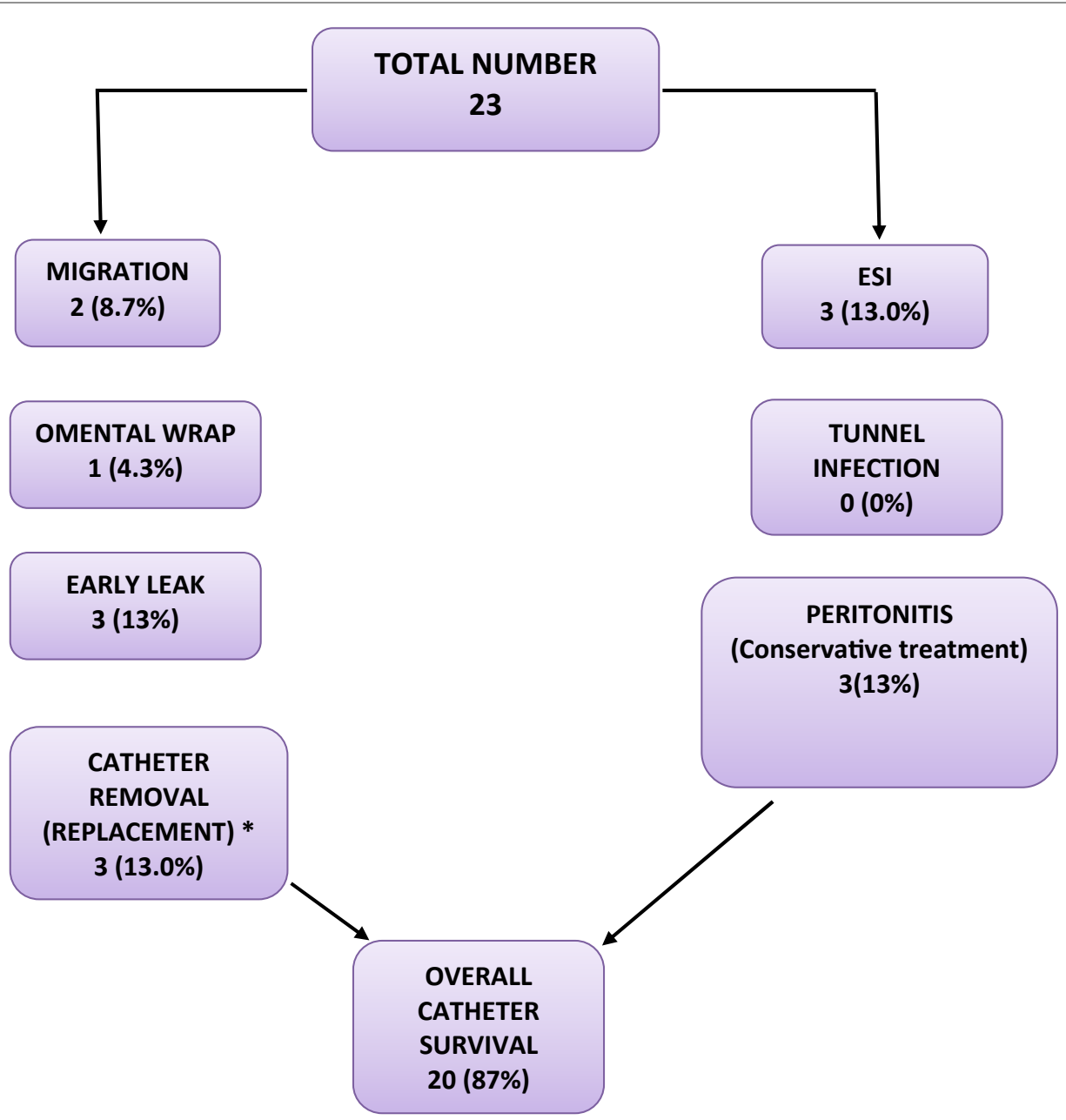

Figure 1: Consort diagram demonstrating the outcome of PD.

*Catheter removal because of technical complications

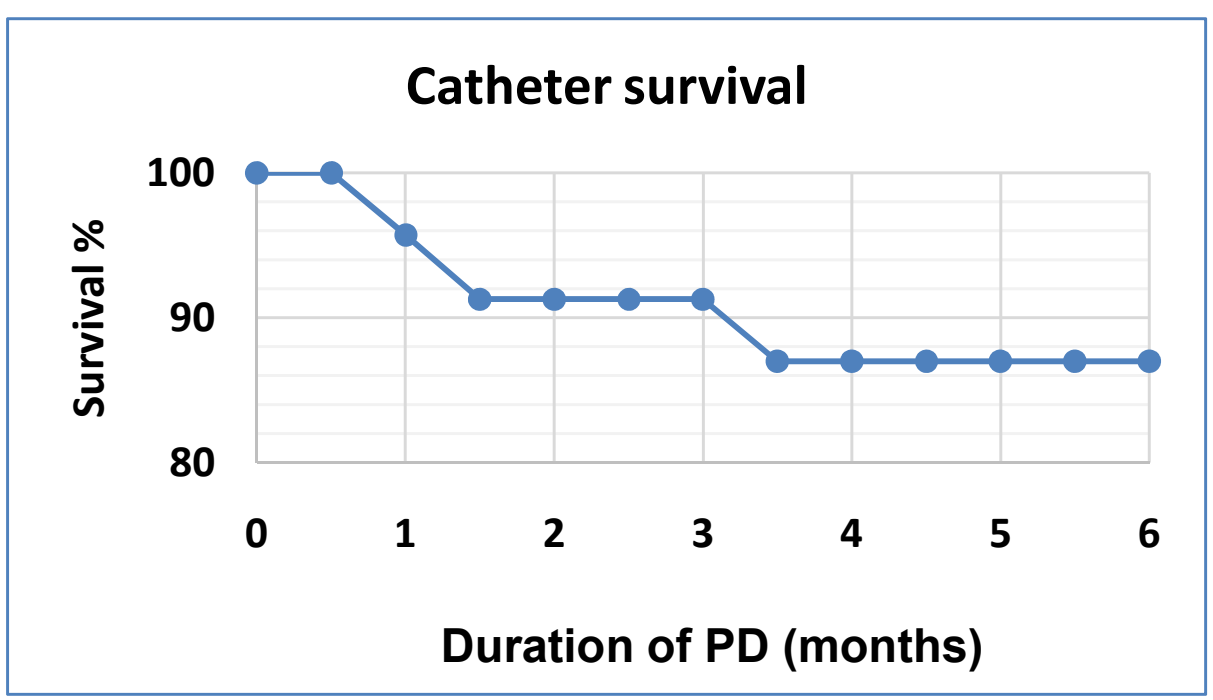

Figure 2: PD catheter survival over 180 days. 
Table 3: Summary of failed vascular access in patients' population.

\begin{tabular}{|c|c|c|c|}
\hline No. & Failed access & No. & Failed access \\
\hline 1 & Rt \& Lt IJV, Rt \& Lt RCF, Rt BCF, Lt BAG, Lt SFA to CFVG & 13 & Rt \& Lt IJV, Rt SCV, Rt BCF, Lt BACVG, Rt \& Lt SFA to CFVG \\
\hline 2 & Rt \& Lt IJV, Rt RCF, Lt BACVG, Rt \& Lt PFVC, Rt BCG & 14 & $\begin{array}{l}\text { RT SCV, Lt IJV, Rt \& Lt RCF, Rt \& Lt BCF, Lt BAG, Lt BBVG, Lt SFA to } \\
\text { CFVG, Rt PFVC }\end{array}$ \\
\hline 3 & $\begin{array}{l}\text { Rt IJV, Lt SCV, Rt \& Lt BCF, Rt BBVG, Lt BTBG, Rt SFA to CFVG, Lt } \\
\text { PFVC }\end{array}$ & 15 & Rt IJV, Lt SCV, Rt \& Lt BCF, Rt BBVG, Lt BTBG, Rt SFA to CFV, Lt PFVC \\
\hline 4 & Rt \& Lt IJV, Rt \& Lt RCF, Rt BCF, Lt BAG, Lt SFA to CFVG & 16 & Rt \& Lt IJV, Rt SCV, Rt BCF, Lt BACVG, Rt \& Lt SFA to CFVG \\
\hline 5 & Rt \& Lt IJV, Rt \& Lt PFVC, Lt BCG, Rt BAG, Rt SFA to CFVG & 17 & Rt IJV, Lt SCV, Rt \& Lt BCF, Rt BBVG, Lt BTBG, Rt SFA to CFV, Lt PFVC \\
\hline 6 & $\begin{array}{l}\text { Rt IJV, RT SCV, Lt IJV, Rt \& Lt RCF, Rt \& Lt BCF, Lt BAG, Lt BBVG, Lt } \\
\text { SFA to CFVG, Rt PFVC }\end{array}$ & 18 & $\begin{array}{l}\text { RT SCV, Lt IJV, Rt \& Lt RCF, Rt \& Lt BCF, Lt BAG, Lt BBVG, Lt SFA to } \\
\text { CFVG, Rt PFVC }\end{array}$ \\
\hline 7 & Rt IJV, Lt SCV, Rt \& Lt BCF, Rt BBVG, Lt BTBG, Rt SFA to CFV, Lt PFVC & 19 & $\begin{array}{l}\text { Rt \& Lt RCF, Rt \& Lt BCF, Rt \& Lt IJV, Rt PFVC, Lt SFA to CFV, Lt BAG, } \\
\text { Rt BACVG }\end{array}$ \\
\hline 8 & Rt IJV, Lt SCV, Lt BCF, Rt \& Lt BACVG, Rt \& Lt SFA to CFV & 20 & Rt \& Lt IJV, Rt RCF, Rt \& Lt BCF, Rt \& Lt BAG, Rt \& Lt SFA to CFV \\
\hline 9 & Rt \& Lt IJV, Rt \& Lt RCF, Rt BCF, Lt BAG, Lt SFA to CFV & 21 & $\begin{array}{l}\text { Rt IJV, Lt SCV, Rt \& Lt BCF, Rt BBVG, Lt BTBG, Rt SFA to CFVG, Lt } \\
\text { PFVC }\end{array}$ \\
\hline 10 & Rt \& Lt RCF, Rt \& Lt RCF, Rt \& Lt IJV, Rt PFVC, Lt SFA to CFV, Lt BAG & 22 & $\begin{array}{l}\text { Rt \& Lt RCF, Rt \& Lt BCF, Rt \& Lt IJV, Rt PFVC, Lt SFA to CFV, Lt BAG, } \\
\text { Rt BACVG }\end{array}$ \\
\hline 11 & $\begin{array}{l}\text { Rt PFVC, Lt SFA to CFV, Rt IJV, Lt IJV, Rt, PFVC, Lt RCF, Rt RCG, Lt } \\
\text { BACVG, Rt BCG }\end{array}$ & 23 & Rt IJV, Lt SCV, Rt \& Lt BCF, Rt BBVG, Lt BTBG, Rt SFA to CFV, Lt PFVC \\
\hline 12 & $\begin{array}{l}\text { Rt IJV, Lt SCV, Rt \& Lt BCF, Rt BBVG, Lt BTBG, Rt SFA to CFVG, Lt } \\
\text { PFVC, }\end{array}$ & & \\
\hline
\end{tabular}

IJV: Internal Jugular Vein permicath; RCF: RadioCephalic Fistula; RCG: RadioCephalic Graft; BCF: BrachioCephalic Fistula; BCG: BrachioCephalic Graft; BAG: BrachioAxillary Graft; SCV: SubClavian Vein access; PFVC: Femoral Vein Permicath; BBVG: Brachial Artery to Basilic Vein Graft; SFA to CFV: Superficial Femoral Artery to Common Femoral Vein; BACVG: Brachial to Antecubital Vein Graft; BTBG: Brachial to Transposed Basilic Vein Fistula.

Table 4: Metabolic control in patients treated with instant-start, life-saving PD.

\begin{tabular}{|c|c|c|c|c|c|c|}
\hline & Pre-PD & After $\mathbf{1}^{\text {st }}$ session & After $2^{\text {nd }}$ session & After $3^{\text {rd }}$ session & After $4^{\text {th }}$ session & $p$ \\
\hline BUN, mg/dl & \multirow{2}{*}{$93.2 \pm 15.6$} & \multirow{2}{*}{$88.5 \pm 15.2$} & \multirow{2}{*}{$76.4 \pm 16.3$} & \multirow{2}{*}{$64.6 \pm 13.5$} & \multirow{2}{*}{$52.3 \pm 10.7$} & \multirow{2}{*}{0.0024} \\
\hline Median \pm SD & & & & & & \\
\hline Creatinine, mg/dl & \multirow{2}{*}{$10.7 \pm 3.1$} & \multirow{2}{*}{$9.4 \pm 3.3$} & \multirow{2}{*}{$9.2 \pm 3.3$} & \multirow{2}{*}{$8.4 \pm 2.5$} & \multirow{2}{*}{$6.7 \pm 2.4$} & \multirow{2}{*}{0.0031} \\
\hline Median \pm SD & & & & & & \\
\hline Potassium, mEq/L & \multirow{2}{*}{$6.3 \pm 0.8$} & \multirow{2}{*}{$5.7 \pm 0.6$} & \multirow{2}{*}{$5.5 \pm 0.4$} & \multirow{2}{*}{$4.7 \pm 0.3$} & \multirow{2}{*}{$4.2 \pm 0.3$} & \multirow{2}{*}{0.0151} \\
\hline Median \pm SD & & & & & & \\
\hline Calcium, mg/dl* & \multirow{2}{*}{$7.6 \pm 2.0$} & \multirow{2}{*}{$7.9 \pm 2.2$} & \multirow{2}{*}{$8.2 \pm 2.1$} & \multirow{2}{*}{$8.8 \pm 2.7$} & \multirow{2}{*}{$8.9 \pm 2.5$} & \multirow{2}{*}{0.0101} \\
\hline Median \pm SD & & & & & & \\
\hline Phosphorous, mg/dl & \multirow{2}{*}{$9.3 \pm 2.7$} & \multirow{2}{*}{$9.1 \pm 2.5$} & \multirow{2}{*}{$8.4 \pm 2.2$} & \multirow{2}{*}{$7.6 \pm 2.2$} & \multirow{2}{*}{$5.5 \pm 1.9$} & \multirow{2}{*}{0.0068} \\
\hline Median \pm SD & & & & & & \\
\hline Bicarbonate, $\mathrm{mEq} / \mathrm{L}$ & \multirow{2}{*}{$16.5 \pm 3.8$} & \multirow{2}{*}{$18.4 \pm 3.9$} & \multirow{2}{*}{$20.2 \pm 3.9$} & \multirow{2}{*}{$21.3 \pm 4.2$} & \multirow{2}{*}{$22.5 \pm 4.3$} & \multirow{2}{*}{0.0044} \\
\hline Median \pm SD & & & & & & \\
\hline
\end{tabular}

PD: Peritoneal Dialysis; BUN: Blood Urea Nitrogen

*Corrected calcium in relation to serum albumin

leak and migration of about $12 \%$ with urgent-start peritoneal dialysis. Their definition of urgent-start PD was when dialysis started within 2 weeks of catheter insertion. They concluded that urgent started peritoneal dialysis has acceptable early complication rate and similar long-term technique survival. Ranganathan $\mathrm{D}$, et al. conducted a randomized controlled study to determine the appropriate time to initiate peritoneal dialysis after catheter insertion (TIMELY STUDY). All catheters were inserted by open surgical approach and the high rate of catheter leakage in this study may be related to the technique of catheter insertion [18]. Bitencourt D, et al. had reported their experience with urgent-start PD in 51 patients, their definition of early start was within 72 hours of catheter insertion. All catheters were inserted percutaneously by nephrologists. They reported catheter displacement in $15.5 \%$, leakage in $7.8 \%$ and peritonitis as 0.5 episode/ patient/year [19]. In a recent publication, $\mathrm{Ye} \mathrm{H}$, et al. [33] reported their 10 -year experience with urgent start PD. The study was a novel one with a large cohort of patients and the authors defined "urgent start" as PD that started within 14 days of catheter insertion. In comparison, we initiated the instant-start, life-saving PD in acutely ill dialysis ESVAF patients within few hours (1-3 h) of patient's presentation. 
Our PD catheters were all inserted by a well-trained nephrology team and our results were far better than what was reported before. In our study, catheter migration occurred in only $8.7 \%$, early leakage in $13 \%$ and technical catheter survival was $87 \%$. In addition, the infectious complications in our study were better than the previously reported figures with peritonitis rate of 0.142 /patient-year and patients' survival of $91.3 \%$ at 180 days.

Our data could have been more interesting if a larger cohort has been documented, however, it has the merit of being the first of its kind that discussed the instant-start PD (within few hours) in a certain group of patients who without our approach may have faced devastating outcomes.

\section{Conclusions}

The instant-start PD modality was a life-saving, feasible and safe alternative to hemodialysis in patients with ESVAF.

\section{Study Limitations}

Although our report is the first of its kind to address instantstart, life-saving PD in this group of ESRD patients with ESVAF, the excellent results we observed with our approach may reflect this center's experience with PD in general and may not be generalized to other centers lacking their expertise.

\section{Disclosures}

The authors have no financial conflicts of interest to declare.

\section{References}

1. United States Renal Data System (2015) USRDS annual data report: Epidemiology of kidney disease in the United States. National Institutes of Health, National Institute of Diabetes and Digestive and Kidney Disease, Bethesda, USA.

2. Pippias $M$, Kramer A, Noordzij M, Afentakis N, de la Torre RA, et al. (2017) The European Renal Association-European Dialysis and Transplant Association Registry Annual Report 2014: a summary. Clin Kidney J 10: 1-16.

3. Lok CE, Foley R (2013) Vascular access morbidity and mortality: trends of the last decade. Clin J Am Soc Nephrol 8: 1213-1219.

4. Valliant A, McComb K (2015) Vascular access monitoring and surveillance: An Update. Adv Chronic Kidney Dis 22: 446-452.

5. Roy-Chaudhury P, Sukhatme VP, Cheung AK (2006) Hemodialysis vascular access dysfunction: a cellular and molecular viewpoint. J Am Soc Nephrol 17: 1112-1127.

6. Dhingra RK, Young EW, Hulbert-Shearon TE, Leavey SF, Port FK (2001) Type of vascular access and mortality in U.S. hemodialysis patients. Kidney Int 60: 1443-1451.

7. Al Shakarchi J, Nath J, McGrogan D, Khawaja A, Field M, et al. (2015) End-stage vascular access failure: can we define and can we classify? Clin Kidney J 8: 590-593.

8. Vascular Access 2006 Work Group (2006) Clinical practice guidelines for vascular access. Am J Kidney Dis 48: S176-S247.

9. Almasri J, Alsawas M, Mainou M, Mustafa RA, Wang Z, et al. (2016) Outcomes of vascular access for hemodialysis: A systematic review and meta-analysis. J Vasc Surg 64: 236-243.

10. Guo A, Mujais S (2003) Patient and technique survival on peritoneal dialysis in the United States: evaluation in large incident cohorts. Kidney Int Suppl 88: S3-S12.
11. Van Biesen W, Dequidt C, Vijt D, Vanholder R, Lameire N (1998) Analysis of the reasons for transfers between hemodialysis and peritoneal dialysis and their effect on survivals. Adv Perit Dial 14: 90-94.

12. Chaudhary K, Sangha H, Khanna R (2011) Peritoneal dialysis first: Rationale. Clin J Am Soc Nephrol 6: 447-456.

13. Heaf JG, Lokkegaard H, Madsen M (2002) Initial survival advantage of peritoneal dialysis relative to hemodialysis. Nephrol Dial Transplant 17: 112-117.

14. Dahlan RA, Alsuwaida AO, Farrash MS, Qureshi MA, Hejaili F, et al. (2017) Let us Listen to Patients: Underutilization of peritoneal dialysis from patients' perspectives. Perit Dial Int 37: 574-583.

15. Alkatheeri AM, Blake PG, Gray D, Jain AK (2016) Success of UrgentStart Peritoneal Dialysis in a Large Canadian Renal Program. Perit Dial Int 36: 171-176.

16. Povlsen JV, Ivarsen P (2006) How to start the late referred ESRD patient urgently on chronic APD. Nephrol Dial Transplant 21: ii56ii59.

17. See EJ, Cho Y, Hawley CM, Jaffrey LR, Johnson DW (2017) Early and late patient outcomes in urgent-start peritoneal dialysis. Perit Dial Int 37: 414-419.

18. Ranganathan D, John GT, Yeoh E, Williams N, O'Loughlin B, et al. (2017) A randomized controlled trial to determine the appropriate time to initiate peritoneal dialysis after insertion of catheter (Timely PD Study). Perit Dial Int 37: 420-428.

19. Bitencourt Dias D, Mendes ML, Burgugi Banin V, Barretti $P$, Ponce D (2017) Urgent-start peritoneal dialysis: The first year of Brazilian experience. Blood Purif 44: 283-287.

20. Gameiro J, Fonseca JA, Jorge S, Lopes JA (2018) Management of end-stage vascular access failure patients: A retrospective analysis. Port J Nephrol Hypert 32: 324-330.

21. Al-Hwiesh AK (2014) Percutaneous peritoneal dialysis catheter insertion by a nephrologist: a new, simple and safe technique. Perit Dial Int 34: 204-211.

22. Al-Hwiesh AK, Abdul-Rahman I, Fenkelstein F, Divino-Filho J, Qutub H, et al. (2018) Acute kidney injury in critically ill patients: A prospective randomized study of tidal peritoneal dialysis versus continuous renal replacement therapy. Ther Apher Dial 22: 371-379.

23. Lee SM, Nam HS, Jeong EG, Son YK, Kim SE, et al. (2014) Comparison of exit site infection and peritonitis incidences between povidoneiodine and normal saline use for chronic exit-site care in peritoneal dialysis patients. Kidney Res Clin Pract 33: 144-149.

24. Hoen B, Paul-Dauphin A, Hestin D, Kessler M (1998) EPIBACDIAL: A multicenter prospective study of risk factors for bacteremia in chronic hemodialysis patients. J Am Soc Nephrol 9: 869-876.

25. Jaar BG, Plantinga LC, Crews DC, Fink NE, Hebah N, et al. (2009) Timing, causes, predictors and prognosis of switching from peritoneal dialysis to hemodialysis: A prospective study. BMC Nephrol 10.

26. Cho Y, See EJ, Htay H, Hawley CM, Johnson DW (2018) Early peritoneal dialysis technique failure: Review. Perit Dial Int 38: 319327.

27. See EJ, Johnson DW, Hawley CM, Pascoe EM, Badve SV, et al. (2018) Risk predictors and causes of technique failure within the first year of peritoneal dialysis: An Australian and New Zealand Dialysis and transplant Registry (ANZDATA) study. Am J Kidney Dis 72: 188-197. 
28. Davies SJ (2001) Peritoneal dialysis in the patient with a failing renal allograft. Perit Dial Int 21: S280-S284.

29. Madar H, Korzets A, Ori Y, Herman M, Levy-Drummer RS, et al. (2010) Residual renal function in peritoneal dialysis after renal transplant failure. Perit Dial Int 30: 470-474.

30. Panocchia N, Tazza L, Di Stasio E, Liberatori M, Vulpio C, et al. (2016) Mortality in hospitalized chronic kidney disease patients starting unplanned urgent hemodialysis. Nephrology (Carlton) 21: 62-67.

31. Povlsen JV, Sorensen $A B$, Ivarsen $P$ (2015) Unplanned start on peritoneal dialysis right after PD catheter implantation for older people with end-stage renal disease. Perit Dial Int 35: 622-624.

32. KDIGO (2013) Clinical practice guideline for the evaluation and management of chronic kidney disease. Kidney Int Suppl 3: S19-S75.

33. Ye H, Yang X, Yi C, Guo Q, Li Y, et al. (2019) Urgent start peritoneal dialysis for patients with end stage renal disease: a 10-year retrospective study. BMC Nephrol 20: 238. 